\title{
RT Crucis: a look into the X-ray emission of a peculiar symbiotic star
}

\author{
L. Ducci ${ }^{1,2}$, V. Doroshenko ${ }^{1}$, V. Suleimanov ${ }^{1,3}$, M. Nikołajuk $^{4,2}$, A. Santangelo ${ }^{1}$, and C. Ferrigno ${ }^{2}$ \\ ${ }^{1}$ Institut für Astronomie und Astrophysik, Kepler Center for Astro and Particle Physics, Eberhard Karls Universität, Sand 1, \\ 72076 Tübingen, Germany \\ e-mail: ducci@astro.uni-tuebingen.de \\ 2 ISDC Data Center for Astrophysics, Université de Genève, 16 chemin d'Écogia, 1290 Versoix, Switzerland \\ 3 Kazan Federal University, Kremlevskaja str., 18, 420008 Kazan, Russia \\ 4 Wydział Fizyki, Uniwersytet w Białymstoku, Ciołkowskiego 1L, 15-245 Białystok, Poland
}

Received 3 February 2016 / Accepted 31 May 2016

\begin{abstract}
Symbiotic stars are a heterogeneous class of interacting binaries. Among them, RT Cru has been classified as prototype of a subclass that is characterised by hard X-ray spectra that extend past $\sim 20 \mathrm{keV}$. We analyse $\sim 8.6 \mathrm{Ms}$ of archival INTEGRAL data collected during the period 2003-2014, 140 ks of Swift/XRT data, and a Suzaku observation of $39 \mathrm{ks,} \mathrm{to} \mathrm{study} \mathrm{the} \mathrm{spectral} \mathrm{X-ray} \mathrm{emission} \mathrm{and}$ investigate the nature of the compact object. Based on the 2MASS photometry, we estimate the distance to the source of 1.2-2.4 kpc. The X-ray spectrum obtained with Swift/XRT, JEM-X, IBIS/ISGRI, and Suzaku data is well fitted by a cooling flow model modified by an absorber that fully covers the source and two partially covering absorbers. Assuming that the hard X-ray emission of RT Cru originates from an optically thin boundary layer around a non-magnetic white dwarf, we estimated a mass of the white dwarf of $M_{\mathrm{WD}} \approx 1.2 M_{\odot}$. The mass accretion rate obtained for this source might be too high for the optically thin boundary layer scenario. Therefore we investigate other plausible scenarios to model its hard X-ray emission. We show that, alternatively, the observed X-ray spectrum can be explained with the X-ray emission from the post-shock region above the polar caps of a magnetised white dwarf with mass $M_{\mathrm{WD}} \approx 0.9-1.1 M_{\odot}$.
\end{abstract}

Key words. X-rays: binaries - white dwarfs - stars: individual: RT Cru - stars: individual: IGR J12349-6434

\section{Introduction}

Symbiotic stars are interacting binaries discovered in the early 1900 's and defined as objects whose optical spectra show bright emission lines (indicating the presence of a hot source) superimposed on a late-type stellar continuum (commonly a M giant; e.g. Allen 1984; Luna et al. 2013). This vague definition makes symbiotic stars a very heterogeneous class of objects. RT Crucis (hereafter RT Cru), which is the subject of this paper, is a member of this class.

RT Cru was discovered as a variable star (Leavitt \& Pickering 1906; Dunér et al. 1911) and later classified as a symbiotic star by Cieslinski et al. (1994). It shows optical flickering in the $V$ and $B$ bands on timescales of $10-30 \mathrm{~min}$ and amplitudes of up to 0.09 mag. Optical flickering has been observed in other symbiotic stars with accreting white dwarfs (WD; e.g. CH Cyg, CI Cyg, T CrB, RS Oph, Cieslinski et al. 1994) and accreting neutron stars (GX 1+4, Braga et al. 1993; Jablonski et al. 1997). Cieslinski et al. (1994) noted that the optical spectrum of RT Cru shows strong similarity to the recurrent nova $\mathrm{T} \mathrm{CrB}$ in quiescence. A search in historical data revealed a large magnitude variation $(\Delta m \sim 1.7$ in Leavitt \& Pickering 1906; $\Delta m \sim 2.4$ in Uiterdijk \& van Houten 1960) compatible with those observed in other symbiotic stars (Cieslinski et al. 1994). Gromadzki et al. (2013) detected two periodicities in ASAS, OGLE, and MACHO lightcurves, $P_{\text {orb }}=325 \pm 9 \mathrm{~d}$ and $P_{\text {puls }}=63 \pm 1 \mathrm{~d}$, interpreted as the orbital period and the pulsation of the red giant. More than a hundred years after its discovery,
RT Cru has recently acquired a significant interest, after the observation of hard X-ray emission detected up to $\sim 150 \mathrm{keV}$ (Chernyakova et al. 2005; Kennea et al. 2009). RT Cru was observed by INTEGRAL in 2003 and 2004 with a 20-60 keV flux of $\approx 4 \times 10^{-11} \mathrm{erg} \mathrm{cm}^{-2} \mathrm{~s}^{-1}$ (Chernyakova et al. 2005) and initially classified as a new source (IGR J12349-6434). Masetti et al. (2005) noted that the position of IGR J12349-6434 was compatible with that of RT Cru. The association between these two sources was later confirmed by Tueller et al. (2005) thanks to the refined position of IGR J12349-6434 obtained with Swift/XRT. RT Cru was detected again with INTEGRAL with a $20-40 \mathrm{keV}$ flux $F_{\mathrm{x}}>4 \times 10^{-11} \mathrm{erg} \mathrm{cm}^{-2} \mathrm{~s}^{-1}$ (Sguera et al. 2012, 2015). The bright, hard X-ray emission properties of RT Cru are shared with a small group of symbiotic stars: T CrB, CD-57 3057, and CH Cyg (Luna \& Sokoloski 2007). In particular, the recurrent nova T $\mathrm{CrB}$, which is believed to be a non-magnetic $1.3 M_{\odot} \mathrm{WD}$ where the hard X-ray emission is produced in the optically thin boundary layer of the accretion disc, has a high X-ray luminosity similar to RT Cru $\left(L_{\mathrm{x}} \approx 10^{34} \mathrm{erg} \mathrm{s}^{-1}\right.$, e.g. Kennea et al. 2009; Luna et al. 2008). Luna \& Sokoloski (2007) reported on a Chandra observation of RT Cru. They proposed that, similarly to $\mathrm{T} \mathrm{CrB}$, the $0.3-8 \mathrm{keV} \mathrm{X}$-ray emission and the flickering variability originates from an optically thin boundary layer around a massive non-magnetic WD powered by an accretion disc. Kennea et al. (2009) studied RT Cru using the data from XRT and BAT instruments on board Swift and suggested that the $\mathrm{X}$-ray variability on timescales of a few days is caused by variable absorption from a clumpy medium moving across the line 
of sight. Eze (2014) studied the iron line complex at 6.4-7 keV of RT Cru with Suzaku. He proposed that the $6.4 \mathrm{keV}$ emission line $(\mathrm{Fe} \mathrm{K} \alpha)$ is caused by the interaction between the hard X-rays and the absorbing material around the system, while the $6.7 \mathrm{keV}$ (Fe XXV) and $7 \mathrm{keV}$ (Fe XXVI) emission lines are produced by photoionization and collisional excitations in the hot plasma in the vicinity of the WD.

In this work we coherently analyse the largest possible set of X-ray data ( $\sim 12$ yrs of archival INTEGRAL data, $\sim 140 \mathrm{ks}$ of Swift/XRT data and $\sim 39 \mathrm{ks}$ of Suzaku observation) to further investigate the properties of RT Cru (Sects. 2 and 3) and clarify the nature of the compact object (Sect. 4).

\section{Observations and data analysis}

\subsection{INTEGRAL}

We analysed archival data of the INTErnational Gamma-Ray Astrophysics Laboratory (INTEGRAL, Winkler et al. 2003) collected with the coded-mask telescope imager on board INTEGRAL satellite (IBIS, Ubertini et al. 2003) and the detector INTEGRAL soft gamma-ray imager (ISGRI, Lebrun et al. 2003). IBIS/ISGRI has a fully-coded field of view of $9^{\circ} \times 9^{\circ}$, a partially coded field of view of $29^{\circ} \times 29^{\circ}$ and operates in the $\sim 15-400 \mathrm{keV}$ energy band. We also used data from the X-ray monitors joint european monitor for X-ray (JEM-X, Lund et al. 2003), which are two co-aligned coded-mask telescopes on board INTEGRAL that operate simultaneously with IBIS/ISGRI in the energy range $3-35 \mathrm{keV}$. Their fields of view have a diameter of $13.2^{\circ}$. Each orbital revolution of INTEGRAL consists of pointings called science windows ( $\mathrm{ScWs).} \mathrm{We} \mathrm{performed} \mathrm{the}$ reduction and data analysis using the off-line science analysis (OSA) 10.2 software provided by the ISDC Data Centre for Astrophysics (Goldwurm et al. 2003; Courvoisier et al. 2003). We analysed the data between 2003 January 29 and 2014 December 20 where RT Cru was within $12^{\circ}$ from the centre of the IBIS/ISGRI field of view because, at larger off-axis angles, the IBIS response is not well known ${ }^{1}$. Data were filtered to exclude bad time intervals. The resulting data set consists of $2880 \mathrm{ScWs}$, corresponding to an exposure time of $8.6 \mathrm{Ms}$.

RT Cru was never detected, being below the $5 \sigma$ threshold of detection, in the individual $22-50 \mathrm{keV}$ sky images of each pointing, while it was detected with a significance of $\sim 30 \sigma$ in the total mosaic image obtained from the combination of the individual images of the whole data set.

We extracted the average JEM-X1 and IBIS/ISGRI spectra from the mosaics using the OSA tool mosaic_spec, which is especially suitable for faint sources. Owing to poor statistics, we did not extract the average JEM-X2 spectrum (the source was observed only in a few pointings). We used the exposureweighted average ancillary response files and the rebinned response matrices specifically generated for the IBIS/ISGRI and JEM-X datasets. Before fitting, we added systematic uncertainties of $2 \%$ and $3 \%$ to the IBIS/ISGRI and JEM-X spectra, respectively.

\subsection{Swift/XRT}

RT Cru was observed with the X-ray Telescope (XRT, Burrows et al. 2005) on board the Swift satellite (Gehrels et al. 2004) since 2005. We collected all the available XRT observations of the target in the time period from 2005 August 20

\footnotetext{
1 See the INTEGRAL data analysis documentation http://www. isdc.unige.ch/integral/analysis
}

23:43:02 UTC to 2012 December 24 10:45:58 UTC (38 observations, total exposure time of $\sim 140 \mathrm{ks}$ ). Part of these data (first six observations) were analysed and the results presented in Kennea et al. (2009). We processed the XRT data obtained in photon-counting (PC) mode with the standard procedures (xrtpipeline v0.13.1) and the calibration files CALDB 4.5.6. Standard grade filtering (0-12) and screening criteria were applied. We checked that no pile-up correction was required. Source counts were accumulated from a circular region with a radius of 20 pixels ( 1 pixel $\sim 2.36^{\prime \prime}$ ) and background counts were accumulated from a circular nearby source-free region at $\mathrm{RA}(\mathrm{J} 2000)=12: 34: 17.84, \operatorname{Dec}(\mathrm{J} 2000)=-64: 32: 08.7$ with a radius of 40 pixels. The spectral energy channels are grouped to have at least 30 counts per bin for good statistical quality of the spectrum.

\subsection{Suzaku}

Suzaku (Mitsuda et al. 2007) observed RT Cru in 2007 July 2 ( $\sim 39 \mathrm{ks})$. We used data from the X-ray imaging spectrometers (XIS, Koyama et al. 2007) and the PIN layer of the hard X-ray detector (HXD Takahashi et al. 2007), which were operated in standard mode during the observation.

The data were processed with the standard Suzaku pipeline included in the HEASOFT software package (version 6.16) and screened according to standard criteria.

XIS events of RT Cru were extracted in each of the three detectors (front-illuminated: XIS 0,3; back-illuminated: XIS 1) in circular regions with radius $2^{\prime}$ centered on the target. Background counts were accumulated from a source-free circular region far from the point-spread function of the source (coordinates of the background region: $\mathrm{RA}(\mathrm{J} 2000)=12: 35: 01.81$, $\operatorname{Dec}(\mathbf{J} 2000)=-64: 41: 56.2$, radius $\left.=2^{\prime}\right)$. Ancillary response and redistribution matrices files for the XIS spectra were generated using the Suzaku tools xisrmfgen and xissimarfgen. For the background subtraction in PIN data, we used the tuned non X-ray background (NXB) models specifically generated for these two observations by the HXD team. For the cosmic X-ray background $(\mathrm{CXB})$, we used the simulated spectrum generated by the Suzaku tool hxdpinxbpi. The PIN response files appropriate for the epoch of the observations and for a point source were selected. The XIS and PIN spectra were rebinned using the optimal binning method of Kaastra \& Bleeker (2016). For the timing analysis we correct the event arrival times to the solar system barycenter with the Suzaku specific tool aebarycen.

\section{Results}

\subsection{Distance}

We compared the published optical/near-infrared spectrum of RT Cru (Fig. 2 of Cieslinski et al. 1994) with those of the spectral atlas of Pickles (1998). Based on the general shape of the spectra, we classified the donor star of RT Cru as an M4III-M5III, which is in agreement with the M4-M5 spectral type obtained by Cieslinski et al. (1994). Since the optical part of the spectrum might be affected by the emission from the compact object companion (Hellier 2001), we estimated the distance using the infrared magnitudes of the 2MASS (Skrutskie et al. 2006) counterpart 2MASS J12345374-6433560. We converted the observed colours and magnitudes to the photometric system of Bessell \& Brett (1988) using the transformation equations 


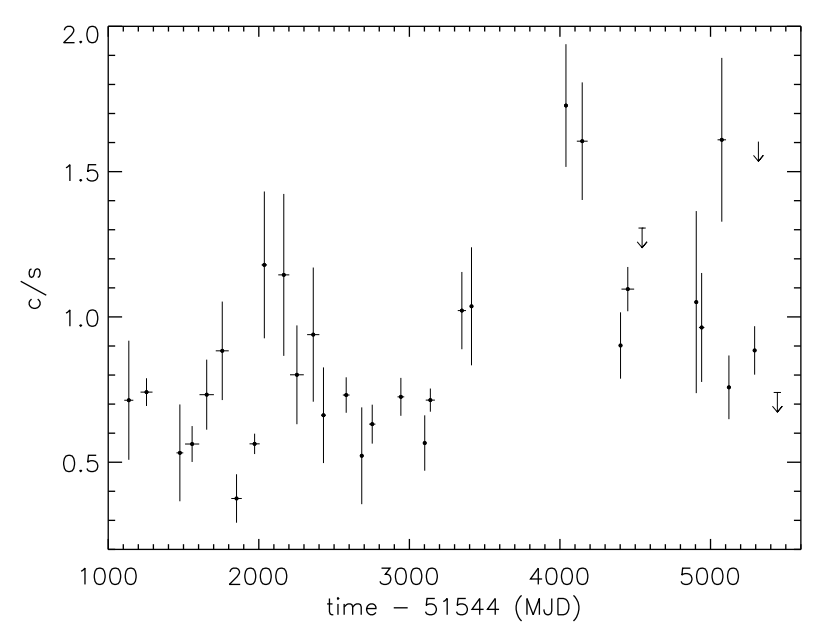

Fig. 1. 22-50 keV IBIS/ISGRI lightcurve. Error bars correspond to $1 \sigma$ confidence level. Downward arrows are $3 \sigma$ upper-limit.

of Carpenter $(2001)^{2}$. Using this information and the intrinsic colours and magnitudes of M4III-M5III stars from Pickles (1998) and Fluks (1998), we calculated the interstellar reddening $E(J-K)$. We obtained the extinction $A_{\lambda}$ (where $\lambda=J, H, K$ ) using the formula of Draine (1989), $A_{\lambda} / E(J-K)=2.4(\lambda / \mu \mathrm{m})^{-\beta}$ (which is valid in the range $0.9<\lambda<6 \mu \mathrm{m}$, and $\beta$ ranges from 1.70 to 1.80 , Draine 2003). M giants show intrinsic variability, so we added a maximum amplitude variation of $\Delta$ mag $=0.25$ (Eyer \& Mowlavi 2008; Tabur et al. 2009) to the 2MASS magnitude uncertainties of RT Cru. We found that the distance of $\mathrm{RT}$ Cru is in the range $\approx 1.2-2.4 \mathrm{kpc}$. This result is in agreement with the value 1.5-2 kpc reported in Luna \& Sokoloski (2007) from a private communication from J. Mikołajewska (2006).

\subsection{Lightcurve analysis}

Figure 1 shows the IBIS/ISGRI lightcurve of RT Cru in the energy band $22-50 \mathrm{keV}$. For each bin (maximum bintime $=100 \mathrm{~d}$ ), we extracted the flux from the mosaic image. RT Cru is always detected with the exception of three bins, where $3 \sigma$ have been reported. The source shows a significant variability resulting in a high value of $\chi^{2}=286.27$ (29 d.o.f.) when the lightcurve is fitted with a constant.

We extracted the Swift/XRT lightcurves of RT Cru in two energy bands, $0.3-4 \mathrm{keV}$ and 4-10 keV. They were corrected for point-spread function losses, vignetting, and background subtracted. Lightcurves are shown in the left panel of Fig. 2, together with their hardness ratio. Each point corresponds to a single observation. Right panel of Fig. 2 shows the hardness ratio as a function of the $0.3-10 \mathrm{keV}$ count rate. The hardness ratio anti-correlates with the $0.3-10 \mathrm{keV}$ rate, as previously noted by Kennea et al. (2009) on a sub-sample of Swift/XRT observations. Kennea et al. (2009) show that the anti-correlation between the flux and hardness ratio is caused by the variability of the intrinsic absorption. We used linear, quadratic, and power-law models to fit the data. The power-law model fits the data better (index $\alpha=-1.09 \pm 0.12$ ). An F-test comparison of the $\chi^{2}$ of the linear and power-law fits gives a probability of chance improvement of the fit of $\sim 1.5 \times 10^{-9}$. The best fit model is plotted in the right

\footnotetext{
2 In particular, we adopted the updated version of the transformation equations of Carpenter (2001), which can be found in: http://www . astro. caltech. edu/ jmc/2mass/v3/transformations/
}

panel of Fig. 2 with a red dashed line. The fitted parameter $\alpha$ is in good agreement with the value obtained by Kennea et al. (2009).

We searched for coherent pulsations in the $0.3-10 \mathrm{keV}$ Swift/XRT lightcurve binned at $5 \mathrm{~s}$ and in the $0.5-10 \mathrm{keV}$ Suzaku lightcurve binned at $8 \mathrm{~s}$, using the fast Lomb-Scargle periodogram technique (Press \& Rybicki 1989). The search was performed on timescales between $10 \mathrm{~s}$ (Swift) and $16 \mathrm{~s}$ (Suzaku) and the time duration of each observation. The number of independent frequencies was estimated using the Eq. (13) of Horne \& Baliunas (1986). We found no evidence for pulsations in the data. We performed simulations on these observations to set a $3 \sigma$ upper-limit on the pulsed fraction of a sinusoidal signal of about $14 \%$ for Swift/XRT and about $8 \%$ for Suzaku.

Luna \& Sokoloski (2007) and Kennea et al. (2009) searched for coherent periodicity in Chandra and Swift/XRT data, but found no evidence for pulsations. The pulsed fraction upperlimits derived by Luna \& Sokoloski (2007) and Kennea et al. (2009) are in agreement with our results.

\subsection{Spectral analysis}

We performed the spectral analysis on two set of data: (1) Swift/XRT data jointly with JEM-X and IBIS/ISGRI data; (2) Suzaku data. We included renormalising constant factors in the spectral fitting to allow for uncertainties between the instruments and differences caused by source variability in nonsimultaneous observations. For the cross-normalisation between Suzaku XIS and PIN, we set the recommended value of 1.18 for observations at the HXD nominal position given in the suzakumemo-2008-06 ${ }^{3}$.

We fitted the spectra with the isobaric cooling flow model (mkcflow in $x$ spec $^{4}$ ), previously used by Luna \& Sokoloski (2007), modified by an absorber that fully covers the source (phabs) and a Gaussian line (gauss) for the $\mathrm{Fe} \mathrm{K} \alpha$ line at $\sim 6.4 \mathrm{keV}$. This model adequately fits the spectra when two partial covering absorbers (pcfabs) are included. In the framework of the "clumpy medium" scenario proposed by Kennea et al. (2009), variable partial covering components take into account the changes in the absorption caused by the clumpy absorbing material around the WD of RT Cru that passes across the line of sight.

The best-fitting parameters are reported in Table 1, with the plot of the spectra with residuals shown in Fig. 3.

We find that the absorption and covering fractions vary between the two data sets, in agreement with the results of Kennea et al. (2009). Similar to what was found by Luna \& Sokoloski (2007), we find that the minimum cooling flow temperature is consistent with the smallest value allowed by the mkcflow model $\left(k T_{\text {min }}=80.8 \mathrm{eV}\right)$, while $k T_{\text {max }}$ from the spectral fit of the Suzaku data is pegged at the largest value allowed by the model $\left(k T_{\max }=79.9 \mathrm{keV}\right)$. The values of the parameter $k T_{\max }$ obtained from the spectral fit of the XRT+INTEGRAL and Suzaku data are different at $90 \%$ confidence level, while they are consistent within a $99 \%$ confidence level. $k T_{\max }$ governs the slope of the spectrum at high energies (the slope increases when $k T_{\max }$ decreases) and the overall height of the spectrum which, however, also depends on the spectral parameter $\dot{M}_{\text {acc }}$. Therefore, $k T_{\max }$ and $\dot{M}_{\text {acc }}$ are correlated, and a higher $k T_{\max }$ in $S u z a k u$ spectrum (whose coverage

\footnotetext{
3 Available at: http://www.astro.isas.jaxa.jp/suzaku/doc/ suzakumemo/suzakumemo-2008-06.pdf

4 All spectral fits have been performed by using xspec v12.8.1g (Arnaud 1996).
} 

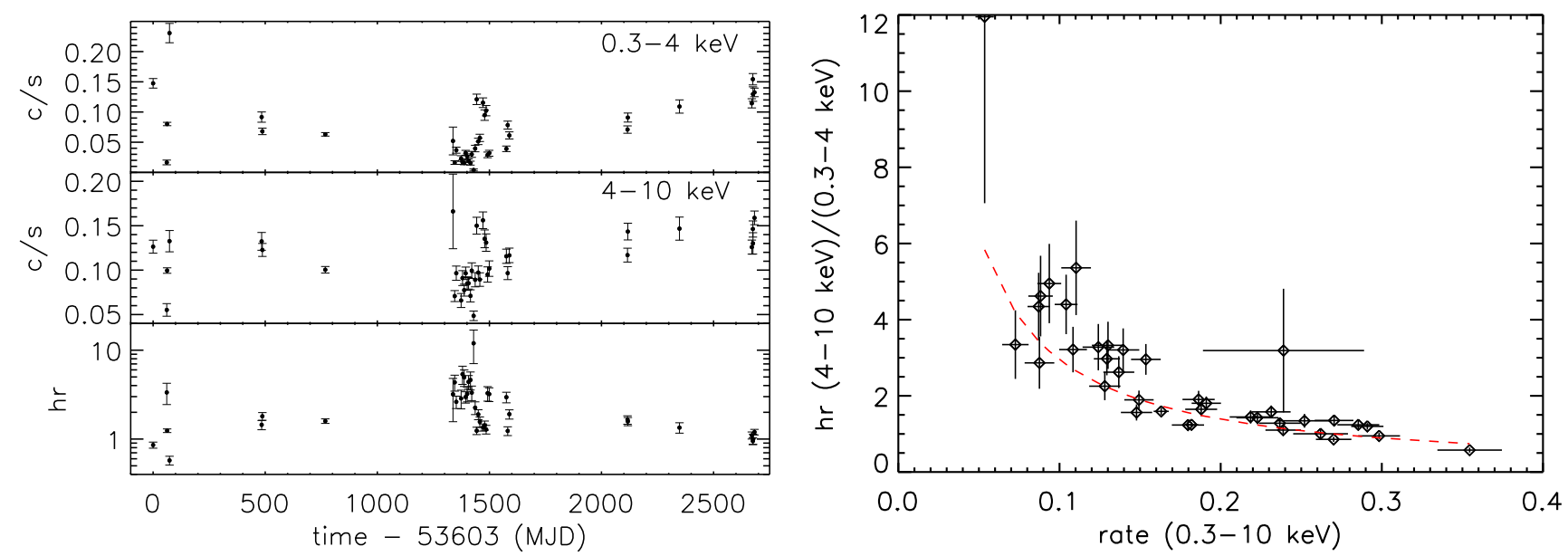

Fig. 2. Left panel: Swift/XRT lightcurves (top: $0.3-4 \mathrm{keV;}$ middle: $4-10 \mathrm{keV}$ ) and hardness ratios as function of time (bottom). Right panel: spectral hardness ratio as function of the $0.3-10 \mathrm{keV}$ count rate. The hardness ratio is the ratio between the $4-10 \mathrm{keV}$ count rate and the $0.3-4 \mathrm{keV}$ count rate. The red dashed line shows the best-fitting power-law model with index $\alpha=-1.09 \pm 0.12$.

Table 1. Best-fitting parameters for the Swift/XRT jointly with the JEM-X and IBIS/ISGRI spectra and Suzaku/XIS+PIN (see Fig. 3).

\begin{tabular}{lcc}
\hline \hline Parameters & INTEGRAL+XRT & Suzaku \\
\hline$N_{\mathrm{H}}$ full covering & \\
$N_{\mathrm{H}}$ partial covering $1^{a}$ & $0.03_{-0.02}^{+0.02}$ & $0.59_{-0.05}^{+0.05}$ \\
covering fraction 1 & $3.01_{-0.09}^{+0.09}$ & $4.2_{-0.6}^{+0.7}$ \\
$N_{\mathrm{H}}$ partial covering $2^{a}$ & $96.4_{-0.8}^{+0.5} \%$ & $70_{-2}^{+2} \%$ \\
covering fraction 2 & $13.4_{-0.5}^{+0.5}$ & $48_{-7}^{+8}$ \\
$k T_{\text {max }}$ & $81.2_{-0.8}^{+0.8} \%$ & $51_{-3}^{+5} \%$ \\
Abundance & $51_{-2}^{+3}$ & $79.9_{-19.9}^{b}$ \\
$\dot{M}_{\text {acc }}$ & $0.51_{-0.15}^{+0.14}$ & $0.53_{-0.09}^{+0.10}$ \\
$E_{\text {Gauss }}(\mathrm{keV})$ & $2.00_{-0.02}^{+0.03}$ & $2.3_{-0.2}^{-0.8}$ \\
$\sigma_{\text {Gauss }}(\mathrm{keV})$ & $6.40 \pm 0.02$ & $6.40^{-0.0} \pm 01$ \\
norm $_{\text {Gauss }}$ & $0.13 \pm 0.02$ & $0.064_{-0.018}^{+0.019}$ \\
$E W_{\text {Gauss }}(\mathrm{eV})$ & $1.35 \pm 0.15 \times 10^{-4}$ & $8.7 \pm 0.9 \times 10^{5}$ \\
$\chi_{v}^{2}($ d.o.f. $)$ & $347_{-46}^{+72}$ & $211_{-22}^{+68}$ \\
\hline
\end{tabular}

Notes. (a) Column density in units of $10^{22} \mathrm{~cm}^{-2}$. (b) Parameter pegged at hard limit; $99 \%$ lower limit: $51 \mathrm{keV} .{ }^{(c)}$ Solar abundance of Anders \& Grevesse (1989). (d) Mass accretion rate in units of $10^{-9} M_{\odot} \mathrm{yr}^{-1}$. Listed uncertainties are at the $90 \%$ confidence level.

at high energies is limited, compared to the INTEGRAL spectrum, up to $\sim 40 \mathrm{keV}$ ) might not necessarily indicate a significant change of the spectral shape at high energies. In fact, when we fit the two spectra with a phenomelogical model, an absorbed power law modified at energies $E>E_{\mathrm{c}}$ with a high-energy cutoff $\left(M(E)=\exp \left[\left(E_{\mathrm{c}}-E\right) / E_{\mathrm{f}}\right]\right.$, highecut in xspec $)$, we find that the shapes of the spectra are consistent within the uncertainties (XRT+INTEGRAL: $\Gamma=0.60_{-0.11}^{+0.10}, E_{\mathrm{c}}=5.2_{-0.5}^{+0.4} \mathrm{keV}$, $E_{\mathrm{f}}=18 \pm 2 \mathrm{keV}$; Suzaku: $\Gamma=0.77_{-0.12}^{+0.17}, E_{\mathrm{c}}=5.1_{-0.7}^{+0.8} \mathrm{keV}$, $E_{\mathrm{f}}=20.1_{-3.3}^{+4.5} \mathrm{keV}$; uncertainties are at the $90 \%$ confidence level). The INTEGRAL spectrum of RT Cru has a better coverage at high energies compared to Suzaku data, hence it is better suited to constrain the parameter $k T_{\max }$.

\section{Discussion}

Pandel et al. (2005) studied the X-ray emission from the boundary layers of ten dwarf novae. They found that the temperature $T_{\max }$ of the cooling flow model is related to the virial temperature $T_{\text {vir }}$, and therefore to the mass of the WD (e.g. Luna \& Sokoloski 2007), by the equation:

$k T_{\max }=\frac{3}{5} k T_{\mathrm{vir}}=\frac{1}{5} \mu m_{\mathrm{p}} v_{\mathrm{K}}^{2}$,

where $v_{\mathrm{K}}$ is the Keplerian velocity, $\mu$ is the mean molecular weight and $m_{\mathrm{p}}$ is the mass of the proton. Assuming that the $\mathrm{X}$-ray emission of RT Cru is produced in the boundary layer of an accretion disc around a non-magnetic WD, we used $k T_{\text {max }}$ obtained from the spectral fit of the INTEGRAL data described in Sect. 3.3, the WD mass-radius relation of Nauenberg (1972), and Eq. (1) to obtain an estimate of WD mass of $M_{\mathrm{WD}} \approx 1.2 M_{\odot}$. This is a refined measurement of the previous estimate obtained by Luna \& Sokoloski (2007; $M_{\mathrm{WD}} \gtrsim 1.3 M_{\odot}$ ) that was based on the $0.3-8 \mathrm{keV}$ Chandra data and therefore was highly uncertain owing to the limited spectral coverage at high energies.

As mentioned above, Luna \& Sokoloski (2007) proposed that the X-ray emission of RT Cru is produced by an optically thin boundary layer of an accretion disc around a massive non-magnetic WD. The mass accretion rate obtained in this work (Sect. 3.3; Table 1), together with the values obtained by Luna \& Sokoloski (2007) and from the formula of the X-ray luminosity of a boundary layer $L_{\mathrm{bl}} \lesssim 0.5\left(G M_{\mathrm{WD}} / R_{\mathrm{WD}}\right) \dot{M}_{\mathrm{acc}}$, is $\dot{M}_{\text {acc }} \approx 2 \times 10^{-9} M_{\odot} \mathrm{yr}^{-1}$. Narayan \& Popham (1993) found that the boundary layer of a $1 M_{\odot}$ WD is expected to be optically thin for $\dot{M}_{\mathrm{acc}} \lesssim 3 \times 10^{-10} M_{\odot} \mathrm{yr}^{-1}$. Therefore, for a high mass-accretion rate like that of RT Cru, the boundary layer is expected to be optically thick and the X-ray spectrum soft, with $k T \lesssim 0.1 \mathrm{keV}$ (Pringle 1977; Frank et al. 2002) ${ }^{5}$. The transition from optically thick to optically thin boundary layer solutions depends on $\dot{M}_{\text {acc }}$, the mass and the rotational angular velocity of the WD, and the viscosity parameter $\alpha$ of the accretion disc (Popham \& Narayan 1995). Although

\footnotetext{
5 Some cataclysmic variables with an optically thick boundary layer show, in addition to the bright soft component, a residual hard X-ray emission whose luminosity and temperature are lower in comparison to those observed when their boundary layers are optically thin (e.g. Wheatley et al. 2003).
} 

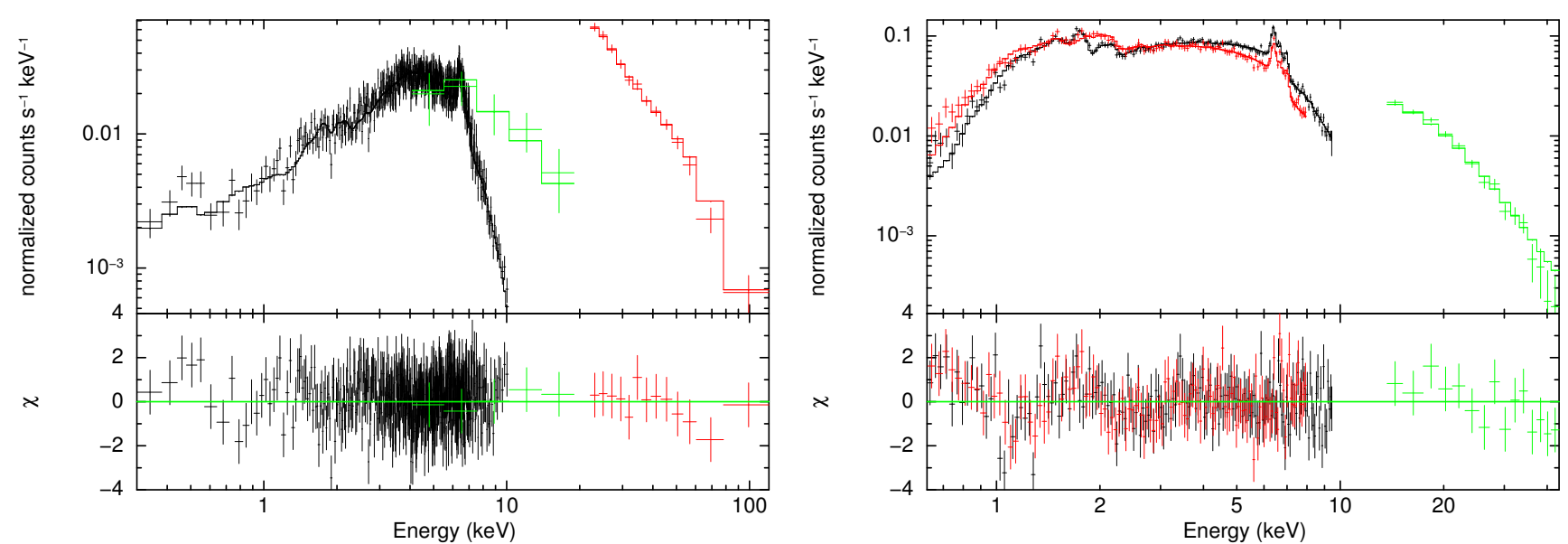

Fig. 3. Left panel: Swift/XRT (black), JEM-X1 (green), IBIS/ISGRI (red) spectrum. Right panel: Suzaku/XIS front-illuminated (black), backilluminated (red), Suzaku/PIN (green). For each panel, the residuals in units of standard deviation are shown. The best-fitting parameters are reported in Table 1.

its exact location in the space of parameters is still not well known, Popham \& Narayan (1995) found that it occurs at larger values of $\dot{M}_{\text {acc }}$ for a more massive WD. On the other hand, Suleimanov et al. (2014) noted that the transition from optically thick to an optically thin boundary layer should occur at lower values of $\dot{M}_{\text {acc }}$ (compared to the results of Popham \& Narayan 1995), if a more realistic coefficient for the Kramers opacity is used. Although the optically thin boundary layer scenario cannot be excluded, its presence in RT Cru is questionable. Therefore, we consider alternative explanations for the hard X-ray emission of RT Cru.

An alternative scenario to explain the observed hard X-ray emission is the accretion onto the polar caps of a magnetised WD. In these objects, the magnetic field is strong enough to disrupt the accretion flow geometry (which can be quasi-spherical or an accretion disc) at some distance from the star surface, and channel the matter onto the polar caps. Shocks form above the polar caps and the hot, optically thin post-shock region (PSR) emits hard X-ray photons. Assuming that the compact object of RT Cru is a magnetic WD, we fitted the XRT+INTEGRAL and Suzaku spectra with the PSR model of Suleimanov et al. (2005) modified by an absorber that fully covers the source, one partial covering absorber, and three narrow Gaussians for the ironline complex. The PSR model of Suleimanov et al. (2005), developed for intermediate polars, enables us to estimate the mass of the WD from the temperature and emissivity distribution in the post-shock region. We fitted the spectra above $3 \mathrm{keV}$, i.e. in the energy range of validity of the model of Suleimanov et al. (2005). The best fits of the spectra give $M_{\mathrm{WD}}=0.93_{-0.07}^{+0.08} M_{\odot}$ for XRT+INTEGRAL data set and $M_{\mathrm{WD}}=1.13_{-0.15}^{+0.15} M_{\odot}$ for Suzaku spectrum (uncertainties are at the $90 \%$ confidence level).

The magnetic WD scenario explains reasonably well the main properties of the X-ray emission of RT Cru. In fact, accreting WDs with strong magnetic fields at the surface $\left(B \sim 10^{6}-10^{7} \mathrm{G}\right)$ are observed at high X-ray luminosities $\left(10^{32}-10^{34} \mathrm{erg} \mathrm{s}^{-1}\right.$; e.g. Sazonov et al. 2006) and with hard $\mathrm{X}$-ray spectra, which can be observed with the currently operating hard X-ray telescopes up to $\sim 100 \mathrm{keV}$ (e.g. Scaringi et al. 2010 and references therein).

The X-ray emission of magnetic WDs is pulsed with typical pulsed fractions in the 40\%-90\% range (Kennea et al. 2009 and references therein). If RT Cru is a magnetic WD, the lack of detection of the pulsation can be explained with a relatively low pulsed fraction of the signal that is caused, for example, by aligned or nearly aligned rotation and magnetic axes. The aligned axes argument was proposed by Ramsay et al. (2008) to explain the lack of detection of coherent X-ray pulsations in V426 Oph and LS Peg, two cataclysmic variables with properties that are similar to intermediate polars. They also suggest that some of the unclassified cataclysmic variables with hard X-ray emission (20-100 keV), which do not show a spin period modulation might be intermediate polars with closely aligned magnetic and spin axes.

\section{Conclusions}

We presented a study of the spectral X-ray emission of RT Cru based on $\sim 8.6 \mathrm{Ms}$ of archival INTEGRAL data, $\sim 140 \mathrm{ks}$ of Swift/XRT data, and a Suzaku observation of 39k. We found that a cooling flow model (mkcflow) modified by an absorber that fully covers the source, two partial covering absorbers, and a Gaussian line for the $\mathrm{Fe} \mathrm{K} \alpha$ line at $\sim 6.4 \mathrm{keV}$ adequately fits the X-ray spectrum. We found that the absorption components are variables, in agreement with the "clumpy medium" scenario proposed by Kennea et al. (2009). So far, the origin of the hard X-ray emission of RT Cru has been explained with an optically thin boundary layer around a non-magnetic WD. In this framework, we used the relation between the $k T_{\max }$ temperature and the mass of the WD of Pandel et al. (2005) to refine the previous measurement of the mass of the WD obtained by Luna \& Sokoloski (2007) to the new value of $M_{\mathrm{WD}} \approx$ $1.2 M_{\odot}$. Since the mass accretion rate might be too high for the optically thin boundary layer scenario, we have shown that another plausible scenario is that the compact object is a magnetic WD, with the hard X-ray emission produced from the postshock region above the polar caps. Using the spectral model of Suleimanov et al. (2005), we estimated a mass of the WD of 0.9-1.1 $M_{\odot}$. Furthermore, we used 2 MASS photometry to estimate the distance to the source of $1.2-2.4 \mathrm{kpc}$.

Acknowledgements. We thank the anonymous referee for constructive comments, which helped to improve the paper considerably. This work is supported by the Bundesministerium für Wirtschaft und Technologie through the Deutsches Zentrum für Luft und Raumfahrt (grant FKZ 50 OG 1602). V.D. thanks the Deutsches Zentrums für Luft und Raumfahrt (DLR) for financial support (grant DLR 50 OR 0702). V.S. thanks DFG for financial support (grant WE 1312/48-1). This work is partially supported by the Polish NCN grant 2012/04/M/ST9/00780. This paper is based on data from observations with 
INTEGRAL, an ESA project with instruments and science data centre funded by ESA member states (especially the PI countries: Denmark, France, Germany, Italy, Spain, and Switzerland), Czech Republic and Poland, and with the participation of Russia and the USA. This research has made use of data obtained from the Suzaku satellite, a collaborative mission between the space agencies of Japan (JAXA) and the USA (NASA).

\section{References}

Allen, D. A. 1984, Ap\&SS, 99, 101

Anders, E., \& Grevesse, N. 1989, Geochim. Cosmochim. Acta, 53, 197

Arnaud, K. A. 1996, in Astronomical Data Analysis Software and Systems V, eds. G. H. Jacoby, \& J. Barnes, ASP Conf. Ser., 101, 17

Bessell, M. S., \& Brett, J. M. 1988, PASP, 100, 1134

Braga, J., Jablonski, F. F., Elizalde, F., \& Steiner, J. E. 1993, Rev. Mex. Astron. Astrofis., 26, 113

Burrows, D. N., Hill, J. E., Nousek, J. A., et al. 2005, Space Sci. Rev., 120, 165

Carpenter, J. M. 2001, AJ, 121, 2851

Chernyakova, M., Courvoisier, T. J.-L., Rodriguez, J., \& Lutovinov, A. 2005, ATel, 519, 1

Cieslinski, D., Elizalde, F., \& Steiner, J. E. 1994, A\&AS, 106, 243

Courvoisier, T. J.-L., Walter, R., Beckmann, V., et al. 2003, A\&A, 411, L53

Draine, B. T. 1989, in Infrared Spectroscopy in Astronomy, ed. E. Böhm-Vitense, ESA SP, 290, 93

Draine, B. T. 2003, ARA\&A, 41, 241

Dunér, N. C., Hartwig, E., \& Müller, G. 1911, Astron. Nachr., 190, 57

Eyer, L., \& Mowlavi, N. 2008, J. Phys. Conf. Ser., 118, 012010

Eze, R. N. C. 2014, MNRAS, 437, 857

Fluks, M. A. 1998, Ph.D. Thesis, University of Amsterdam, The Netherlands

Frank, J., King, A., \& Raine, D. J. 2002, Accretion Power in Astrophysics, 3rd edn. (Cambridge University Press)

Gehrels, N., Chincarini, G., Giommi, P., et al. 2004, ApJ, 611, 1005

Goldwurm, A., David, P., Foschini, L., et al. 2003, A\&A, 411, L223

Gromadzki, M., Mikołajewska, J., \& Soszyński, I. 2013, Acta Astron., 63, 405

Hellier, C. 2001, Cataclysmic Variable Stars (New York: Springer)

Horne, J. H., \& Baliunas, S. L. 1986, ApJ, 302, 757

Jablonski, F. J., Pereira, M. G., Braga, J., \& Gneiding, C. D. 1997, ApJ, 482, L171
Kaastra, J. S., \& Bleeker, J. A. M. 2016, A\&A, 587, A151

Kennea, J. A., Mukai, K., Sokoloski, J. L., et al. 2009, ApJ, 701, 1992

Koyama, K., Tsunemi, H., Dotani, T., et al. 2007, PASJ, 59, 23

Leavitt, H. S., \& Pickering, E. C. 1906, Harvard College Observatory Circular, 120,1

Lebrun, F., Leray, J. P., Lavocat, P., et al. 2003, A\&A, 411, L141

Luna, G. J. M., \& Sokoloski, J. L. 2007, ApJ, 671, 741

Luna, G. J. M., Sokoloski, J. L., \& Mukai, K. 2008, in RS Ophiuchi (2006) and the Recurrent Nova Phenomenon, eds. A. Evans, M. F. Bode, T. J. O'Brien, \& M. J. Darnley, ASP Conf. Ser., 401, 342

Luna, G. J. M., Sokoloski, J. L., Mukai, K., \& Nelson, T. 2013, A\&A, 559, A6 Lund, N., Budtz-Jørgensen, C., Westergaard, N. J., et al. 2003, A\&A, 411, L231

Masetti, N., Bassani, L., Bird, A. J., \& Bazzano, A. 2005, ATel, 528, 1

Mitsuda, K., Bautz, M., Inoue, H., et al. 2007, PASJ, 59, 1

Narayan, R., \& Popham, R. 1993, Nature, 362, 820

Nauenberg, M. 1972, ApJ, 175, 417

Pandel, D., Córdova, F. A., Mason, K. O., \& Priedhorsky, W. C. 2005, ApJ, 626, 396

Pickles, A. J. 1998, PASP, 110, 863

Popham, R., \& Narayan, R. 1995, ApJ, 442, 337

Press, W. H., \& Rybicki, G. B. 1989, ApJ, 338, 277

Pringle, J. E. 1977, MNRAS, 178, 195

Ramsay, G., Wheatley, P. J., Norton, A. J., Hakala, P., \& Baskill, D. 2008, MNRAS, 387, 1157

Sazonov, S., Revnivtsev, M., Gilfanov, M., Churazov, E., \& Sunyaev, R. 2006, A\&A, 450, 117

Scaringi, S., Bird, A. J., Norton, A. J., et al. 2010, MNRAS, 401, 2207

Sguera, V., Bird, A. J., \& Sidoli, L. 2015, ATel, 8448

Sguera, V., Drave, S. P., Sidoli, L., et al. 2012, ATel, 3887, 1

Skrutskie, M. F., Cutri, R. M., Stiening, R., et al. 2006, AJ, 131, 1163

Suleimanov, V., Hertfelder, M., Werner, K., \& Kley, W. 2014, A\&A, 571, A55

Suleimanov, V., Revnivtsev, M., \& Ritter, H. 2005, A\&A, 435, 191

Tabur, V., Kiss, L. L., \& Bedding, T. R. 2009, ApJ, 703, L72

Takahashi, T., Abe, K., Endo, M., et al. 2007, PASJ, 59, 35

Tueller, J., Gehrels, N., Mushotzky, R. F., et al. 2005, ATel, 591, 1

Ubertini, P., Lebrun, F., Di Cocco, G., et al. 2003, A\&A, 411, L131

Uiterdijk, J., \& van Houten, C. J. 1960, Annalen van de Sterrewacht te Leiden, 21, 355

Wheatley, P. J., Mauche, C. W., \& Mattei, J. A. 2003, MNRAS, 345, 49

Winkler, C., Courvoisier, T. J.-L., Di Cocco, G., et al. 2003, A\&A, 411, L1 